\title{
Novel Coronavirus Disease 2019 and Subarachnoid Hemorrhage: A Case Report
}

\author{
Alexandra Craen ${ }^{1}$, Gideon Logan ${ }^{1}$, Latha Ganti ${ }^{2,}$ 3, 4 \\ 1. Emergency Medicine, University of Central Florida College of Medicine, Orlando, USA 2. Emergency Medicine, \\ Envision Physician Services, Nashville, USA 3. Emergency Medicine, University of Central Florida College of \\ Medicine/Hospital Corporation of America Graduate Medical Education Consortium of Greater Orlando, Orlando, USA \\ 4. Emergency Medical Services, Polk County Fire Rescue, Bartow, USA
}

Corresponding author: Latha Ganti, latha.ganti@ucf.edu

\begin{abstract}
The novel coronavirus disease 2019 (COVID-19) has had a profound impact on healthcare systems around the world. The emergency department (ED) in particular has become the frontline for the identification and care of these patients. While its effects on respiratory symptoms are well recognized, neurologic manifestations have been rarer. We report the case of a patient who presented in cardiac arrest with the return of spontaneous circulation (ROSC). The patient was found to have subarachnoid hemorrhage and later tested positive for COVID-19.
\end{abstract}

Categories: Emergency Medicine, Neurology

Keywords: covid-19, subarachnoid hemorrhage

\section{Introduction}

The novel coronavirus was identified in December of 2019 after an outbreak of pneumonia cases in Wuhan, China. It spread rapidly and was declared a pandemic by the World Health Organization on March 11, 2020 [1]. The disease was designated as COVID-19, caused by the virus termed as severe acute respiratory syndrome coronavirus 2 (SARS-CoV-2) [2]. It has been confirmed in over a million people worldwide at this time. The most common symptoms include fever, cough, and dyspnea, with initial reports from China showing only mild symptoms in $81 \%$ of patients. Critical symptoms of the disease, including respiratory failure and shock, were reported in $5 \%$ of patients. The overall fatality rate associated with the disease is reported to be $2.3 \%$ [3]. Other symptoms reported from around the world include smell and taste abnormalities and gastrointestinal symptoms $[4,5]$. Neurologic symptoms and intracranial hemorrhages have rarely been reported to date.

In this report, we present the case of a patient who was brought in by paramedics as a case of cardiac arrest with the return of spontaneous circulation (ROSC) after recent respiratory symptoms. The patient was subsequently found to have a subarachnoid hemorrhage and also tested positive for COVID-19.

Received 04/14/2020

Review began 04/17/2020 Review ended 04/17/2020 Published 04/27/2020

\section{() Copyright 2020}

Craen et al. This is an open access article distributed under the terms of the Creative Commons Attribution License CC-BY 4.0., which permits unrestricted use, distribution, and reproduction in any medium, provided the original author and source are credited.

\section{Case Presentation}

A 66-year-old female with a past medical history of diabetes, hypertension, and hyperlipidemia was brought to the emergency department (ED) post-cardiac arrest with ROSC. The patient had a one-week history of dry cough, shortness of breath, and general malaise. She had just returned from a month-long stay in the Dominican Republic five days prior to the onset of symptoms. She had not had any sick contacts within her travel group or where she had been staying while out of the country. On the day of the presentation to the ED, the patient's son had found her on the floor next to her bed sitting upright. She had asked him to take her to the ED because of difficulty in breathing and he had called an ambulance. Before the paramedics had arrived, the patient had become unresponsive and stopped breathing. Her son had immediately started cardiopulmonary resuscitation (CPR). Paramedics had arrived on the scene about five minutes after the onset of bystander CPR and had found the patient to be in asystole. They had achieved ROSC after two administrations of epinephrine and seven additional minutes of CPR. The paramedics had informed us of COVID-19 risk factors prior to the arrival, and we wore appropriate personal protective equipment during patient encounters.

On arrival to the ED, the patient was ventilated through a supraglottic airway with a right tibial intraosseous line in place. The patient again had a cardiac arrest on arrival with a pulseless electrical activity (PEA) rhythm noted on the monitor. She had multiple arrests during the initial presentation and an additional 11 minutes of CPR was administered. She received epinephrine, sodium bicarbonate, and calcium chloride during these resuscitations. The patient was intubated using video laryngoscopy and an emergent femoral central line was placed. She was started on a norepinephrine drip after the final ROSC in the ED. The patient was placed on a ventilator on volume control with positive end-expiratory pressure (PEEP) of $12 \mathrm{cmH} 2 \mathrm{O}$. Arterial blood gas after intubation showed a pH of 6.78, pCO2 of 77.5, and a pO2 of 97 on 100\% FiO2. A sodium bicarbonate drip was initiated. A chest X-ray showed bilateral infiltrates (Figure 1). 


\section{Cureus}

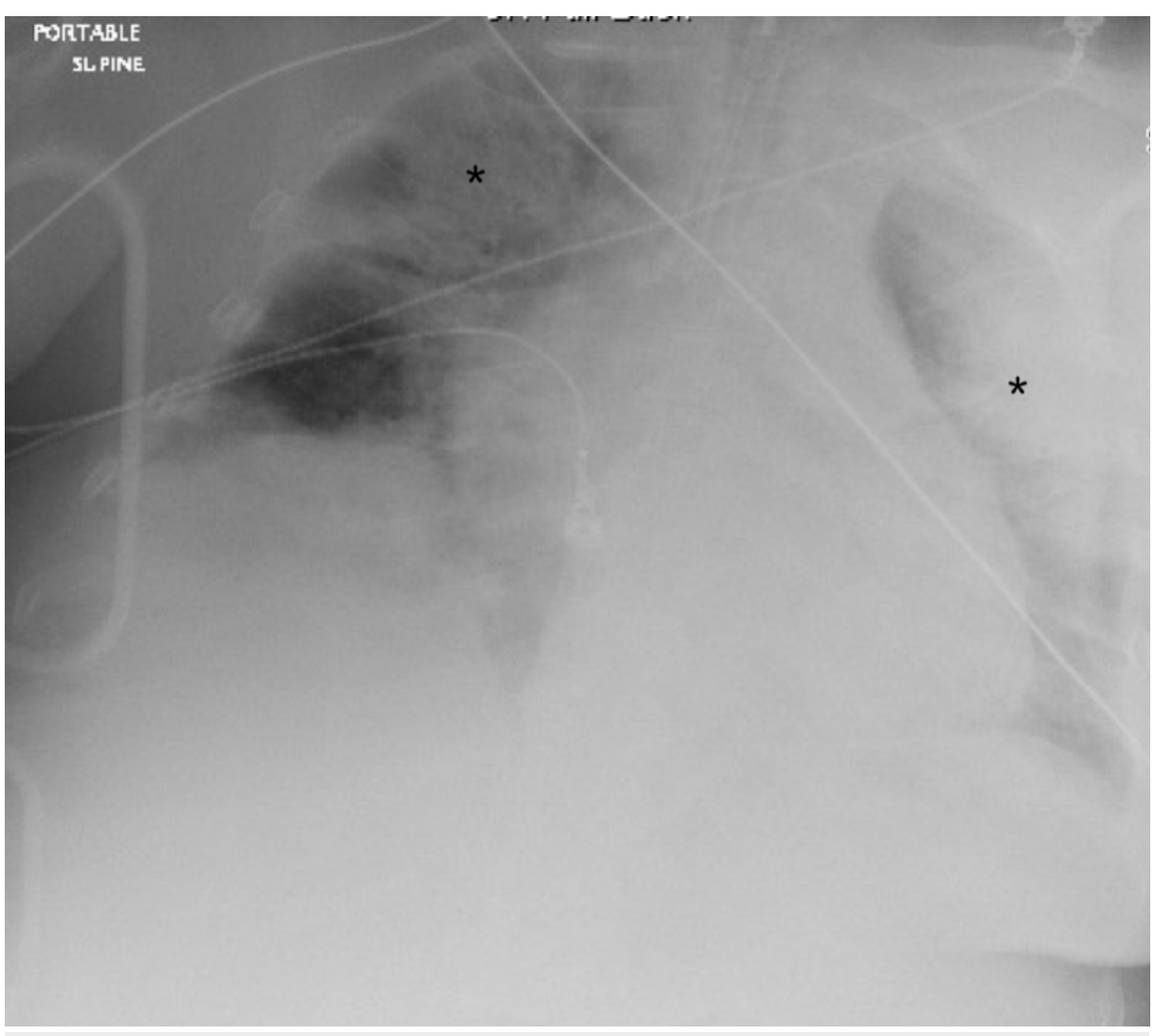

FIGURE 1: Chest X-ray showing bilateral infiltrates (asterisks)

The patient was started on a sepsis protocol with intravenous vancomycin and cefepime. Given the preceding respiratory symptoms and imaging, a swab for SARS-CoV-2 was sent. The patient was not started on sedation in the ED as she remained unresponsive on examination. Initial labs showed elevated creatinine, troponin, and liver enzymes consistent with her cardiac arrest and shock. Her international normalized ratio (INR) was 1.2 and her platelets were within normal limits. Her white blood cells were elevated and her lymphocytes were within normal limits. Her lactic acid was $20.5 \mathrm{mmol} / \mathrm{L}$. Her influenza testing was negative.

The intensive care team was consulted, but the patient had to remain in the ED due to a lack of bed availability in the intensive care unit (ICU). Approximately five hours after the initial intubation, the patient's oxygen saturation dropped to around $80 \%$ on the ventilator and a chest X-ray revealed a $30 \%$ rightsided pneumothorax (Figure 2). 


\section{Cureus}

FIGURE 2: Chest X-ray demonstrating 30\% pneumothorax on the right (arrow)

A 24-French chest tube was placed with the resolution of the pneumothorax. CT of the chest revealed a resolving right anterior pneumothorax with extensive bilateral ground-glass opacifications, considered a pulmonary hallmark for COVID-19 infection (Figure 3) [6].

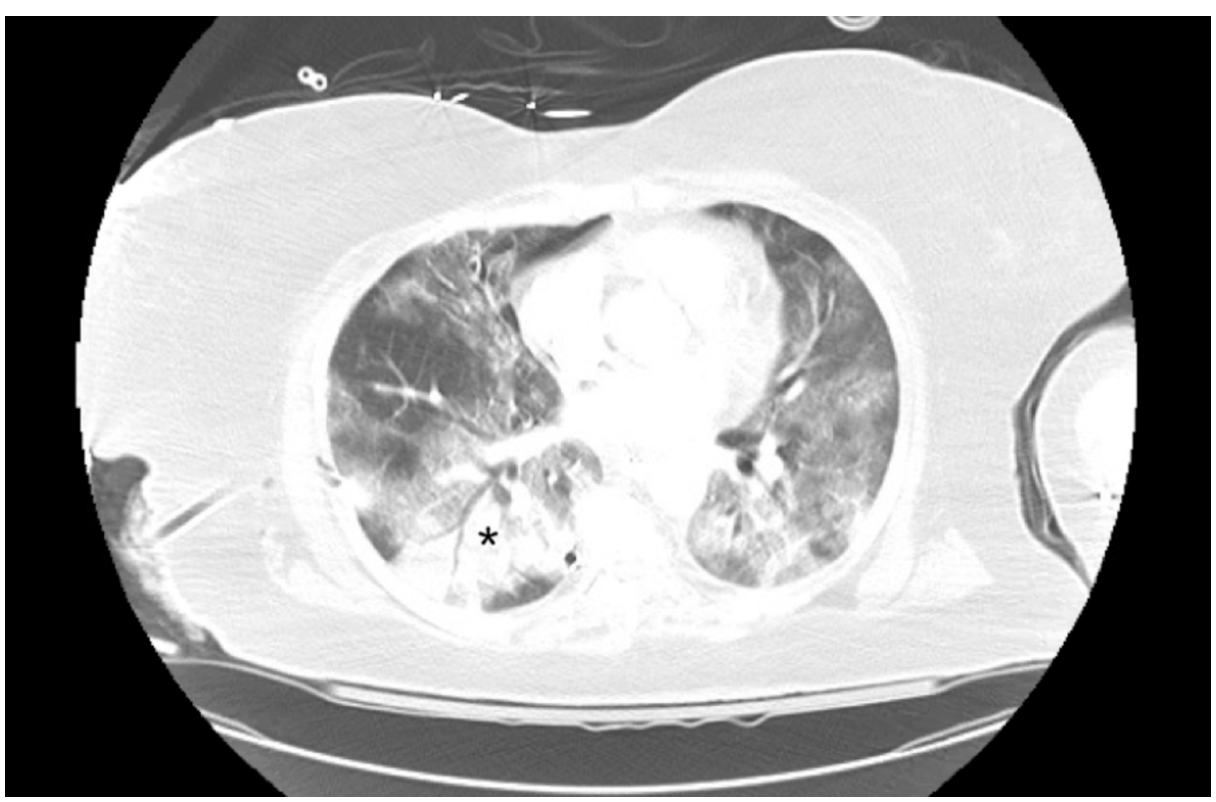

FIGURE 3: Chest CT demonstrating bilateral ground-glass opacities (asterisk), a pulmonary hallmark of COVID-19 infection

CT: computed tomography 


\section{Cureus}

CT imaging of the brain revealed extensive subarachnoid hemorrhage extending into the suprasellar cistern, Sylvian and interhemispheric fissures, effacement of the fourth ventricle, and diffuse cerebral edema (Figure 4).

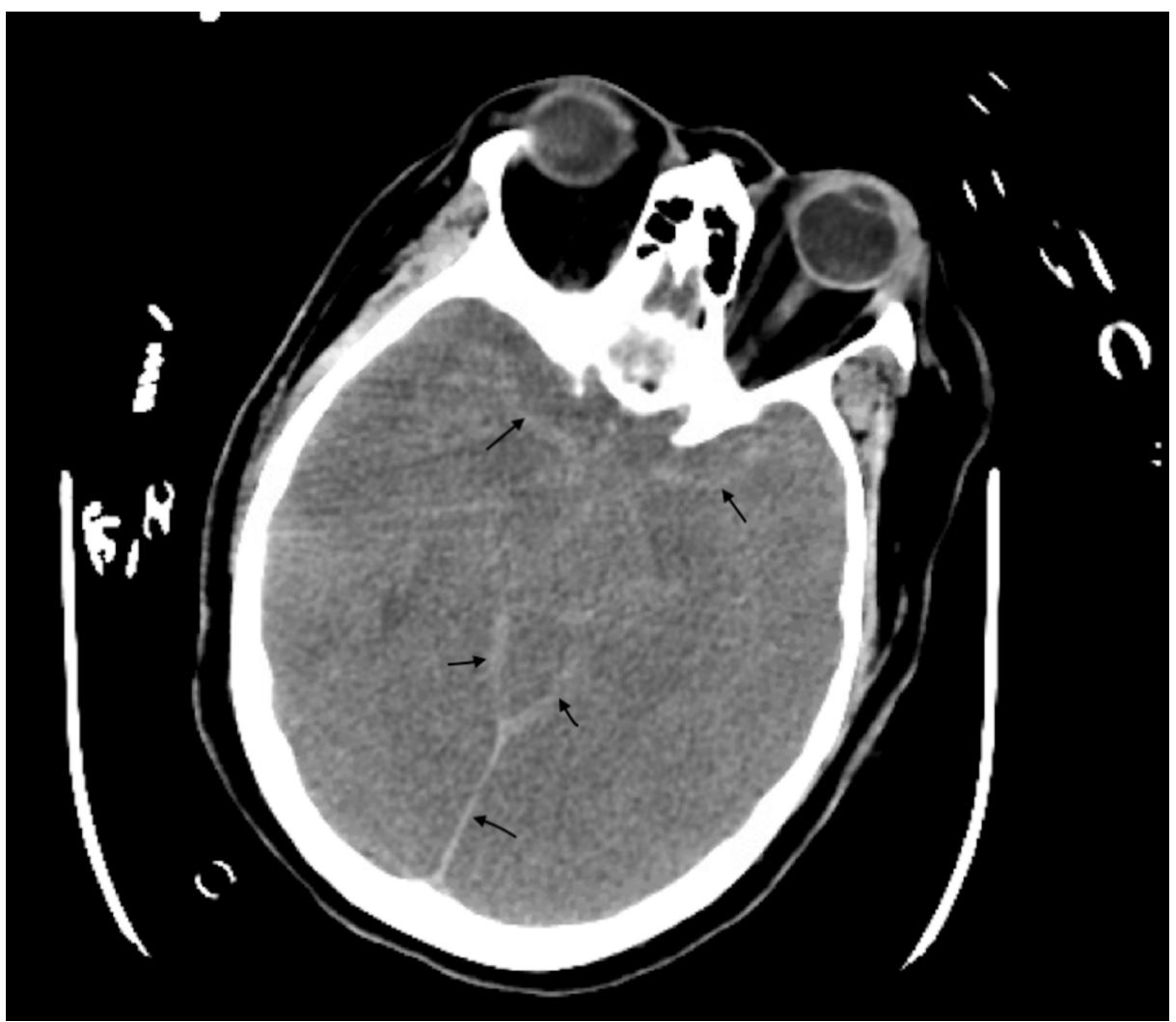

FIGURE 4: Brain CT demonstrating extensive subarachnoid hemorrhage (arrows)

CT: computed tomography

A CT of the cervical spine was negative for fracture. Neurosurgery was consulted but no emergent surgical interventions were recommended given the poor neurological exam. She was given mannitol by the intensive care team and then started on hypertonic saline. They also started azithromycin and hydroxychloroquine for suspected COVID-19.

In the ICU, the patient was unresponsive to verbal or painful stimuli, had 6-mm fixed pupils nonreactive to light, absent corneal and gag reflexes, and absent oculocephalic and vestibulocephalic movements. A nuclear medicine brain flow scan showed the absence of intracranial cerebral perfusion, which supported the clinical diagnosis of brain death. The patient again had a cardiac arrest the next night and was pronounced dead. Her COVID-19 testing later returned positive.

\section{Discussion}

The diagnosis and management of COVID-19 patients continue to evolve. Understanding the potential manifestations of the disease is an important aspect of early treatment. A study from Wuhan, China reported neurological symptoms in $36.4 \%$ of patients. These were described as acute cerebrovascular accidents including intracranial hemorrhage, impaired consciousness, and muscular injuries. These symptoms were more often noted in patients with severe disease. One out of the 214 patients included in the study were noted to have an intracranial hemorrhage [7].

There is a case report of an adult female with respiratory symptoms who developed altered mental status and was found to have acute hemorrhagic necrotizing encephalitis and positive SARS-CoV-2 testing [8]. They attributed this to a possible intracranial cytokine storm, which has been reported in COVID-19 patients with severe illness (Figure 5) [9]. 


\section{Cureus}

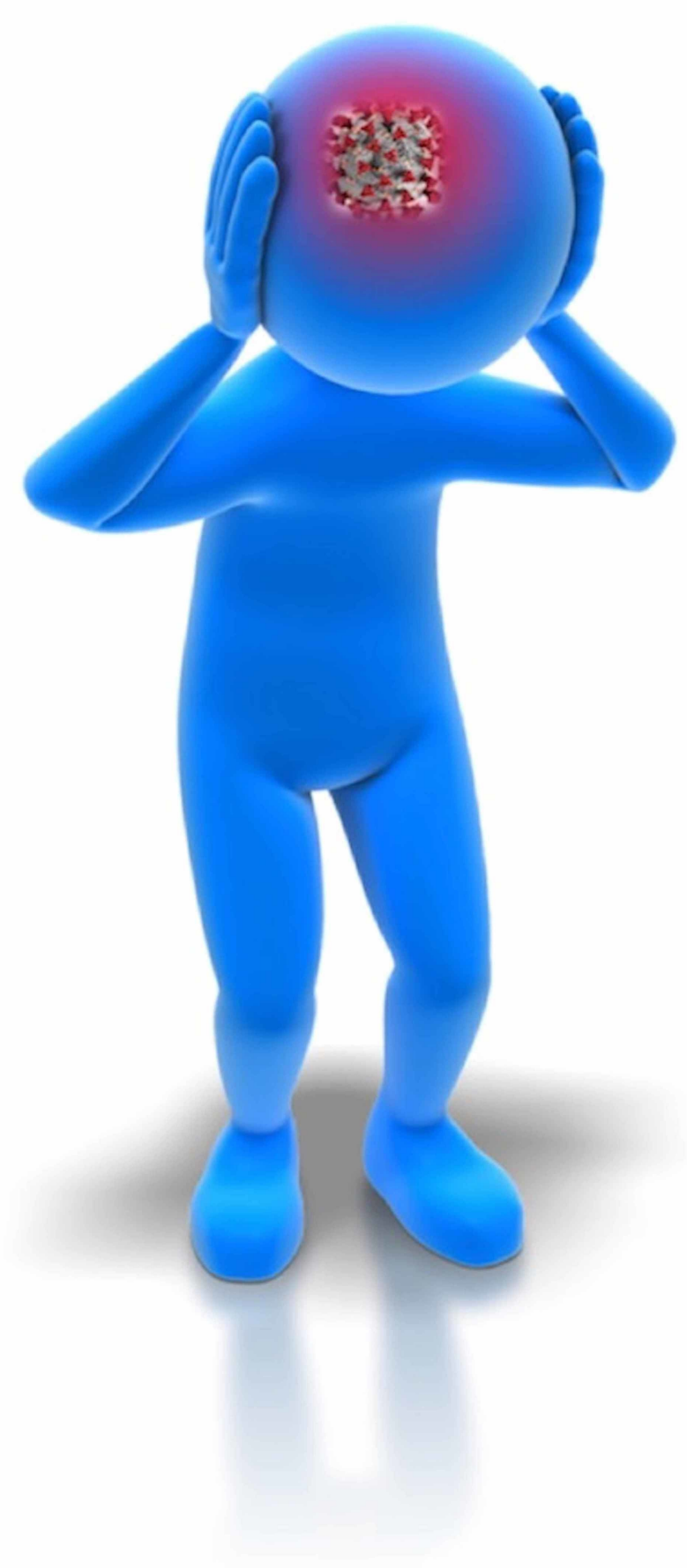

FIGURE 5: Artistic rendering of COVID-19 attacking the brain COVID-19: coronavirus disease 2019

Studies have also noted thrombocytopenia in these patients [5]. A patient in Thailand presented with petechiae only and later tested positive for COVID-19 [10]. A case report prior to this pandemic had linked immune thrombocytopenic purpura (ITP) to coronavirus [11]. However, our patient did not have thrombocytopenia on initial lab tests.

Intracranial hemorrhages appear to be a rare finding associated with COVID-19, with an unknown etiology. Our patient was initially found sitting next to her bed, although there was no reported head trauma or evidence of external trauma on examination. A pseudo-subarachnoid hemorrhage secondary to severe cerebral edema is also possible, although less likely, as evidence of subarachnoid hemorrhage was present on several CT slices [12]. Our patient had a one-week history of respiratory symptoms prior to her cardiac 
arrest and a subsequent diagnosis of subarachnoid hemorrhage. It seems that severe COVID-19 disease could be linked to intracranial hemorrhaging from cytokine storms or coagulation abnormalities. Regardless, it is important to consider severe neurologic disease in COVID-19-positive patients with altered mental status.

\section{Conclusions}

Initially, the presentation of COVID-19 infection was thought to consist of fever, cough, and predominantly respiratory symptoms. However, as this case illustrates, severe neurological manifestations including intracranial hemorrhages should be considered in COVID-19-positive patients with altered mental status.

\section{Additional Information \\ Disclosures}

Human subjects: Consent was obtained by all participants in this study. Hospital Corporation of America Centralized Algorithms for Research Rules on IRB Exemptions (CARRIE)/IRB manager issued approval 2020252. Hospital Corporation of America Centralized Algorithms for Research Rules on IRB Exemptions (CARRIE)/IRB manager issued approval 2020-252. Based on the information provided and attested as true, the research plan described does not require IRB oversight. This is because you are either a) not engaging in research with human subjects as defined by federal regulations; b) engaging in research with human subjects deemed excluded from IRB oversight per 45CFR46.102(1); OR c) engaging in research with sufficient human subject protections in the design to meet one or more IRB exemption criteria set forth in 45CFR46.104. Conflicts of interest: In compliance with the ICMJE uniform disclosure form, all authors declare the following: Payment/services info: All authors have declared that no financial support was received from any organization for the submitted work. Financial relationships: All authors have declared that they have no financial relationships at present or within the previous three years with any organizations that might have an interest in the submitted work. Other relationships: All authors have declared that there are no other relationships or activities that could appear to have influenced the submitted work.

\section{Acknowledgements}

This research was supported (in whole or in part) by HCA Healthcare and/or an HCA Healthcare affiliated entity. The views expressed in this publication represent those of the author(s) and do not necessarily represent the official views of HCA Healthcare or any of its affiliated entities.

\section{References}

1. WHO Director-General's opening remarks at the media briefing on COVID-19: March 16, 2020. (2020). Accessed: April 11, 2020: https://www.who.int/dg/speeches/detail/who-director-general-s-openingremarks-at-the-media-briefing-on-covid-19---16-....

2. WHO: naming the coronavirus disease (COVID-19) and the virus that causes it . (2020). Accessed: April 11, 2020: https:/www.who.int/emergencies/diseases/novel-coronavirus-2019/technical-guidance/naming-thecoronavirus-disease-(co....

3. Wu Z, McGoogan JM: Characteristics of and Important lessons from the coronavirus disease 2019 (COVID19) outbreak in China: summary of a report of 72314 cases from the Chinese Center for Disease Control and Prevention (Epub ahead of print). JAMA. 2020, 10.1001/jama.2020.2648

4. Giacomelli A, Pezzati L, Conti F, et al.: Self-reported olfactory and taste disorders in SARS-CoV-2 patients: a cross-sectional study (Epub ahead of print). Clin Infect Dis. 2020, 10.1093/cid/ciaa330

5. Huang C, Wang Y, Li X, et al.: Clinical features of patients infected with 2019 novel coronavirus in Wuhan, China. Lancet. 2020, 395:497-506. 10.1016/S0140-6736(20)30183-5

6. Kanne JP, Little BP, Chung JH, Elicker BM, Ketai LH: Essentials for radiologists on COVID-19: an updateRadiology Scientific Expert Panel (Epub ahead of print). Radiology. 2020, 200527. 10.1148/radiol.2020200527

7. Mao L, Jin H, Wang M, et al.: Neurological manifestations of hospitalized patients with COVID-19 in Wuhan, China: a retrospective case series study (Epub ahead of print). JAMA Neurol. 2020, 10.1001/jamaneurol.2020.1127

8. Poyiadji N, Shahin G, Noujaim D, Stone M, Patel S, Griffith B: COVID-19-associated acute hemorrhagic necrotizing encephalopathy: CT and MRI features (Epub ahead of print). Radiology. 2020, 201187.

9. Mehta P, McAuley DF, Brown M, Sanchez E, Tattersall RS, Manson JJ: COVID-19: consider cytokine storm syndromes and immunosuppression. Lancet. 2020, 395:1033-34. 10.1016/S0140-6736(20)30628-0

10. Joob B, Wiwanitkit V: Hemorrhagic problem among the patients with COVID-19: clinical summary of 41 Thai infected patients (Epub ahead of print). Clin Appl Thromb Hemost. 2020, 26:10.1177/1076029620918308

11. Magdi M, Rahil A: Severe immune thrombocytopenia complicated by intracerebral haemorrhage associated with coronavirus infection: a case report and literature review. Eur J Case Rep Intern Med. 2019, 6:001155. Accessed: April 26, 2020: https://www.ncbi.nlm.nih.gov/pmc/articles/PMC6663043/. 10.12890/2019_001155

12. Given CA 2nd, Burdette JH, Elster AD, Williams DW 3rd: Pseudo-subarachnoid hemorrhage: a potential imaging pitfall associated with diffuse cerebral edema. AJNR Am J Neuroradiol. 2003, 24:254-6. 